\title{
Unusual reasons and imaging finding in (PRES) posterior reversible encephalopathy syndromes
}

\begin{abstract}
Posterior reversible encephalopathy syndrome (PRES) is a rare clinico-radiological entity characterized by rapid onset of symptoms including headache, seizures, altered consciousness, and visual disturbance. Associated with white matter changes predominantly affecting the posterior parietal and occipital lobes of the brain on neuro imaging. Several conditions have been associated with PRES. The well documented are hypertension; eclampsia, immunosuppressive and cytotoxic treatments, autoimmune diseases and infections/sepsis. There are as well numerous associations rarely or anecdotally reported. It is particularly important not to exclude PRES as a possible diagnosis when we have the appropriate clinical presentation accompanied by the typical radiological findings. It is an increasingly recognized disorder, with a wide clinical spectrum of both symptoms and triggers, and yet it remains poorly understood. We report unusual reasons of PRES and findings.
\end{abstract}

Keywords: PRES, seizures, case Series

\author{
Volume 8 Issue 3 - 2018
}

\begin{abstract}
Aya Imad Shubbar,' Ali R Dehdashtian, ${ }^{2}$ Muhammad Arif, ${ }^{3}$ Khurram A Siddiqui ${ }^{4}$

IInternal Medicine Resident, Department of Academic Affairs, UAE

${ }^{2}$ Consultant Neuroradiologist, Department of Radiology,Al Ain Hospital, UAE

${ }^{3}$ Specialist Internal Medicine/Neurology, Al Ain Hospital, UAE ${ }^{4}$ Consultant Neurologist and Epileptologist, Al Ain Hospital, UAE
\end{abstract}

Correspondence: Dr. Aya Imad Shubbar, Department of Academic Affairs, Internal Medicine Resident, Al Ain Hospital, Al Ain, UAE, Tel +97137022000, Email ashubbar@seha.ae

Received: April 18, 2018| Published: May 08, 2018

\section{Introduction}

Posterior reversible encephalopathy syndrome (PRES) is a rare clinic-radiological entity characterized by rapid onset of symptoms including headache, seizures, altered consciousness, and visual disturbance. Associated with white matter changes predominantly affecting the posterior parietal and occipital lobes of the brain on neuroimaging. Several conditions have been associated with PRES. The well-documented are hypertension, eclampsia, immunosuppressive and cytotoxic treatments, autoimmune diseases and infections/sepsis. There are as well numerous associations rarely or anecdotally reported. It is particularly important not to exclude PRES as a possible diagnosis when we have the appropriate clinical presentation accompanied by the typical radiological findings. It is an increasingly recognized disorder, with a wide clinical spectrum of both symptoms and triggers, and yet it remains poorly understood. We report unusual reasons of PRES ${ }^{1-3}$ and findings. We present three different cases of PRES ${ }^{1-3}$ who have been different presentations and had similar diagnosis.

\section{Material}

We present three cases who have been diagnosed as PRES (Posterior Reversible Encephalopathy Syndrome) with different reasons on MRI scan. There were different types of PRES which were seen on imaging which appear dissolved on seen on imaging.

\section{Case-series}

\section{Case I}

We present a 16-year-old female presented, one week postpartum with seizures. She had normal blood pressure throughout her pregnancy and afterword's had normal pregnancy until she had a seizure. Her neuroimaging showed features of high signal lesion within cortex and subcortical lesion of both parietal and occipital lobes with associated gyral swelling, with no associated gyral restriction. Her neuroimaging showed features of PRES with resolution on repeat scan, see case and Figure1.

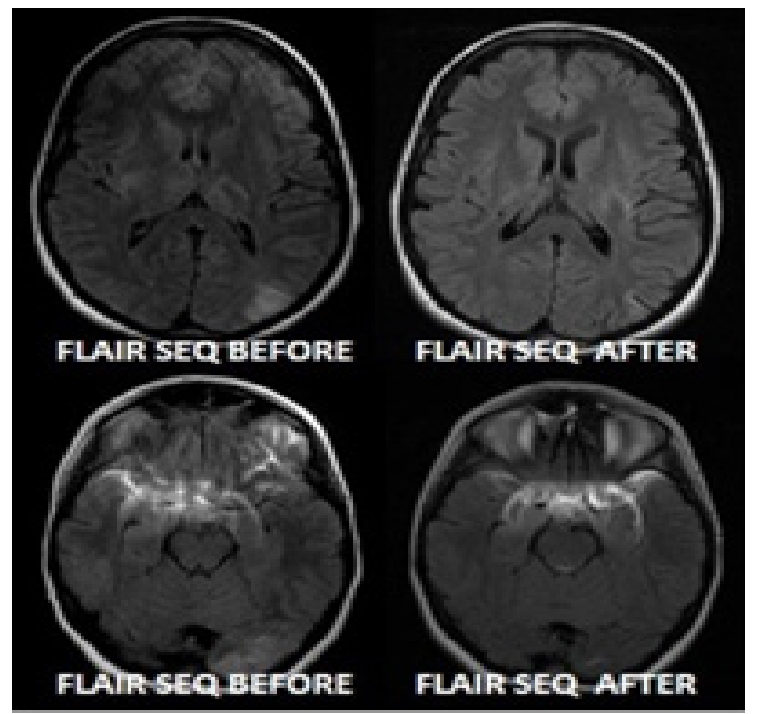

Figure I Image quality degraded by dental braces induced artefacts. There are high signal lesions within the cortex and subcortical region of both parietal and occipital lobes with associated gyral swelling. No associated restricted diffusion. The appearances favor PRES. Follow up images after treatment revealed significant improvement.

\section{Case 2}

A 17-year-old female presented, with three generalized tonic clonic seizure, with preceding abdominal pain (non-surgical) for three days with vomiting. She had autonomic dysfunction with heart rate ranging from $63-120$ beats per minute, with blood pressure ranging from systolic 174-110 and diastolic from 120-80mmHg. Her Urinary 
porphobilinogen and aminolevulinic acid were markedly increased consistent with acute intermittent porphyria and her high signal lesions within the cortex and subcortical region of both hemisphere with associated gyral swelling. No associated restricted diffusion. Her neuroimaging showed features of PRES and favors PRES with resolution on repeat scan. Follow up images after treatment revealed significant improvement, see case and Figure 2.

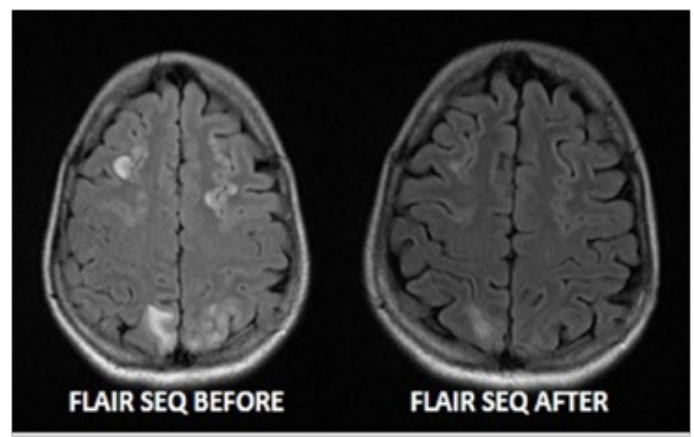

Figure 2 There are high signal lesions within the cortex and subcortical region of both hemispheres with associated gyral swelling. No associated restricted diffusion. The appearances favor PRES. Follow up images after treatment revealed significant improvement.

\section{Case 3}

A 56-year-old female presented, with headache and high blood pressure $>218 / 119 \mathrm{~mm}$ of $\mathrm{Hg}$. Newly discovered renal impairment was seen with Urea of $24.87 \mathrm{mmol} / 1$, and Creatinine of $668.4 \mathrm{mmol} / 1$. Her GCS dropped showed prompted diffuse signal alteration and edema of brain stem. There is bilateral signal alteration seen in the pons and midbrain seen bilaterally, which shows improvement, with bilateral cerebellar peduncle as well as dentate nucleus, not seen in imaging. Diffuse signal alteration and edema of brain stem. DWI images displayed no evidence restriction or acute infarction. Findings are suggestive of hypertensive encephalopathy (Central-Variant PRES). Follow up images after treatment revealed significant improvement. In short MRI Brain which showed central variant of PRES, which shows resolution on repeat scan, see case and Figure 3.

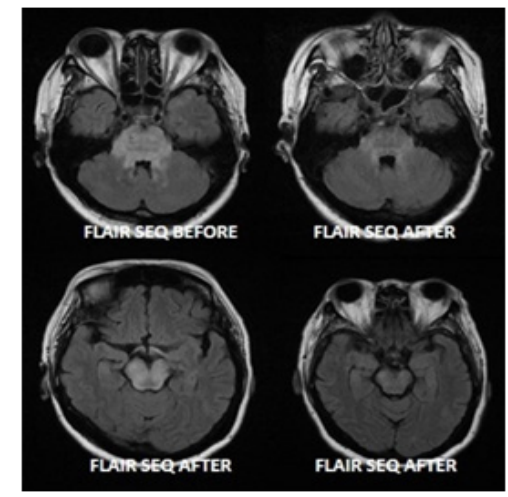

Figure 3 Diffuse signal alteration and edema of brain stem. There is signal alteration of bilateral cerebellar peduncle as well as dentate nucleus (not seen in this image). DWI images displayed no evidence restriction or acute infarction. Findings are suggestive of hypertensive encephalopathy (Central-Variant PRES). Follow up images after treatment revealed significant improvement.

\section{Conclusion}

We present three interesting cases where we could not find reasons for the first case for PRES, although she was post-partum but had no evidence of blood pressure or raised urinary protein to suggest eclampsia or preeclampsia, clinical patient 1 . Our second case eludes to change in blood pressure due to autonomic dysfunction secondary to acute intermittent porphyria which leads to high blood pressure and seizure and imaging showed PRES, clinical patient 2. Out last case suggests uncontrolled blood pressure and renal failure which caused central variant of PRES, clinical patient 3. Post-Partum can be independent risk factors for PRES regardless of eclampsia, blood pressure and urinary protein. ${ }^{1}$ Acute Intermittent Porphyria can present as PRES as imaging feature which can be secondary to autonomic dysfunction. ${ }^{2}$ Central-Variant PRES is rare imaging feature and needs to be recognised. ${ }^{3}$ PRES has been described a un-usual neurological syndrome characterized by a variety of symptoms, including unusual headache, altered mental status, unusual visual disturbances and seizures, and is accompanied by a unique, potentially reversible imaging pattern which are unusual in some cases. Causes of PRES are diverse as described as above of different times and include hypertension, eclampsia/pre-eclampsia, sepsis, immunosuppressive agents, chemotherapy, collagen vascular disease and renal failure. Although the pathophysiology of PRES remains unknown, the currently preferred explanation relates to hypertension, impaired autoregulation and hyperperfusion. ${ }^{4}$ The classic imaging findings are of un-usual vasogenic edema in the subcortical white matter of the parietal and occipital lobes. ${ }^{5}$ As experience with PRES grows in the literature, atypical presentations of PRES are being increasingly described. Authors have demonstrated some cases of PRES with atypical vasogenic edema patterns of distribution, such as frontal lobe, cerebellum, basal ganglia or brain stem involvement. ${ }^{6}$ Some usual cases of unilateral cases of PRES have also been demonstrated. ${ }^{4}$ Knowledge of the different presentations of PRES is important, as atypical imaging findings should not dissuade a diagnosis of PRES in the correctable clinical context. ${ }^{6-8}$ The purpose of these unusual cases which appear as imaging features of atypical PRES. The cases illustrated in this review demonstrate clinical and imaging with atypical presentations characteristic of atypical PRES, with the characteristic clinical and imaging irreversibility associated with this diagnosis along with a diagnosis which makes shores that each case has a diagnosis of atypical PRES which appears as atypical presentation and then appears as the diagnosis needs to be treated. ${ }^{6-8}$

As demonstrated, PRES can present with a variety of imaging findings on CT and MRI. Although originally described as a bilateral symmetrical pattern of vasogenic edema in the posterior parenchyma, other distributions of vasogenic edema can be seen, including a unilateral pattern and involvement of the cerebellum and brain stem. ${ }^{6-}$ ${ }^{8}$ The atypical findings of patchy discontinuous vasoconstriction on angiography are well recognized. Furthermore, atypical presentation, the type and late presentation and is increasingly being recognized in atypical PRES and does not exclude the diagnosis. This can be anatomically located in the brain parenchyma and be late onset focal hematoma or petechial gyral pattern, or in the subarachnoid space, could be the presentation. Restricted diffusion is an atypical finding in PRES, but has been described and should not preclude the diagnosis of PRES in the appropriate clinical context., 


\section{Acknowledgements}

None.

\section{Conflict of interest}

The author declares no conflict of interest.

\section{References}

1. Bartynski WS. Posterior reversible encephalopathy syndrome, part 1 fundamental imaging and clinical features. AJNR Am J Neuroradiol. 2008;29(6):1036-1042.

2. Zhao B, Wei Q, Wang Y, et al. Posterior reversible encephalopathy syndrome in acute intermittent porphyria. Pediatr Neurol. 2014;51(3):457-60.

3. McKinney AM, Jagadeesan BD, Truwit CL. Central-variant posterior reversible encephalopathy syndrome: brainstem or basal ganglia involvement lacking cortical or subcortical cerebral edema. AJR Am J Roentgenol. 2013;201(3):631-638.
4. Bartynksi WS, Boardman JF. Distinct imaging patterns and lesion distribution in posterior reversible encephalopathy syndrome. AJNR Am J Neuroradiol. 2007;28(7):1320-1327.

5. McKinney AM, Short J, Truwit CL, et al. Posterior reversible encephalopathy syndrome: incidence of atypical regions of involvement and imaging findings. AJR Am J Roentgenol. 2007;189(4):904-912.

6. Decker DA, Falchook AD, Yachnis AT, et al. Radiographic and pathologic findings in an atypical brainstem variant of reversible posterior leukoencephalopathy syndrome. Neurologist. 2009;15(6):364-366.

7. Benziada-Boudour A, Schmitt E, Kremer S, et al. Posterior reversible encephalopathy syndrome: a case of unusual diffusion-weighted MR images. J Neuroradiol. 2009;36(2):102-105.

8. Hinchey J, Chaves C, Appignani B, et al. A reversible posterior leukoencephalopathy syndrome. N Engl J Med. 1996;334(8):494-500. 